\title{
Biodecolorization of anthraquinone dyes using immobilised mycelium of Bjerkandera adusta CCBAS930
}

\author{
Kamila Rybczyńska-Tkaczyk ${ }^{1}$, Teresa Korniłłowicz-Kowalska ${ }^{1}$ \\ 1University of Life Sciences in Lublin, Department of Environmental Microbiology, Laboratory of Mycology, \\ Leszczyńskiego 7 Street, 20-069 Lublin, Poland, e-mail:kamila.rybczynska-tkaczyk@up.lublin.pl
}

\begin{abstract}
The aim of this study was to characterize the activity of oxidoreductases during biotransformation of $0.01 \%$ anthraquinone dyes: Alizarin Blue Black B (ABBB) and Acid Blue 129 (AB129), Carminic Acid (CA), Remazol Brilliant Blue R (RBBR), Acid Green 25 (AG25) and Poly R-478 by immobilized strain of Bjerkandera adusta CCBAS 930. Phenolic compounds, phytotoxicity (Lepidium sativum L.), biotoxicity were evaluated to determine the toxicity of anthraquinone dyes before and after the treatment with immobilized B. adusta CCBAS 930. More than 60\% of CA and AB129 were removed by immobilized B. adusta CCBAS after 7 days. No secondary products toxic to plants and bacteria were formed during immobilized cultures of B. adusta CCBAS 930.
\end{abstract}

Keywords: white-rot fungi, versatile peroxidase, anthraquinone dyes, immobilization

\section{Introduction}

Synthetic dyes are widely used in many industries, such as textile, cosmetic, pharmaceutical and food industries. Antraquinone dyes are most widely used for the dyeing of cotton, wool and silk. Textile industry produces the largest amount of dye effluents. In recent years, with the increase in demand for textile products, the global textile production has increased rapidly and reached about 140.84 million tons in 2012 [1]. Currently, wastewater containing textile effluents are treated using physicochemical methods: ozonation, adsorption and membrane filtration [2]. In recent years biological methods using microorganisms to remove contaminants are gaining in importance, supplementing or presenting an alternative to conventional methods. The biological methods are attractive because of their low costs, environmental safety and common societal acceptance. Particularly promising in this regard are white-rot fungi (Basidiomycetes). The most frequently mentioned biological mechanism of contaminant removal by these organisms is biodegradation, involving exploitation of the natural ability of the fungi to synthesize extracellular ligninolytic enzymes. Owing to the low substrate specificity of these enzymes, they can be used for biodegradation of natural and synthetic aromatic compounds that are structurally related to lignin [3]. Decolorization and biodegradation abilities of different Bjerkandera spp., including B. adusta strains, were widely studied, and basically include azo, anthraquinonic, triphenylmethans and heterocyclic dyes. Studies using the anamorphic stage of Bjerkandera adusta CCBAS 930 have shown that this strain can remove a wide spectrum of aromatic compounds from an aquatic environment. In previous studies the B. adusta CCBAS 930 strain has been shown to exhibit decolourizing activity on synthetic dyes with an anthraquinone structure (monoanthraquinones: Carminic Acid and Remazol Brilliant Blue R; polyanthraquinones: Poly-R) [4,5], Alizarin Blue Black B, Acid Blue 129 (data not show), triphenylmethane dyes (Brilliant Green) and heterocyclic dyes (erythrosine) [6]. The use of such immobilized cultures for biodegradation of aromatic compounds has many advantages. Immobilized cells are more stable, durable, and resistant to environmental conditions than conventional cultures $[7,8]$.

The aim of this study was to determine the effectivenesses of antrhraquinone dyes: Alizarin Blue Black B (ABBB), Acid Blue 129 (AB129), Carminic Acid (CA), Remazol Brilliant Blue R (RBBR), Acid Green 25 (AG25) and Poly R-478 decolorization and peroxidases production by immobilised mycelium of B. adusta CCBAS 930.

\section{Materials and Methods}

\subsection{Strain of Bjerkandera adusta CCBAS 930}

Anamorphic stage of $B$. adusta CCBAS 930 was isolated from the black earth soil (Pheozems, FAO classification) from a field near Lublin, South-Eastern Poland [9].

\subsection{Immobilisation of mycelium of $B$. adusta CCBAS 930}


One hundred $\mathrm{ml}$ of a sterile Na-alginate solution (4\%) were mixed with $20 \mathrm{~g}$ of homogenized mycelia of B. adusta CCBAS 930 and its mutants 930-5 and agitated $(150 \mathrm{rpm}, 20 \mathrm{~min})$. The mixture $(10 \mathrm{ml})$ was extruded using a sterile syringe into $0.2 \mathrm{M} \mathrm{CaCl}_{2}$, thus forming beads with a $3.0-4.0 \mathrm{~mm}$ diameter. The beads were allowed to harden in $0.2 \mathrm{M} \mathrm{CaCl}_{2}$ for about $24 \mathrm{~h}$ at $4^{\circ} \mathrm{C}$. Next, the $0.2 \mathrm{M} \mathrm{CaCl}_{2}$ solution was removed and the beads were washed twice with the addition of distilled water. Immobilised mycelium (Na-alginate beads) of $B$. adusta CCBAS 930 was incubated with $0.01 \%$ solutions of anthraquinone dyes $\left(150 \mathrm{rpm}, 28^{\circ} \mathrm{C}\right.$, 7 days).

\subsection{Biochemical analysis of supernatants}

Samples were collected every day and following parameters were estimated: the degree of decolorization of anthraquinone dyes using an UV/VIS spectrophotometer [10] content of phenolic compounds [11] and activity of horseradish-type (HRP-like) and versatile (VP). The activity of HRP-like was estimated using $255 \mu \mathrm{l}$ of $0.01 \%$ o-dianisidine $\left(\varepsilon_{460 \mathrm{~nm}}=11,3 \mathrm{M}^{-1}\right.$ $\mathrm{cm}^{-1}$ ) in $0.1 \mathrm{M}$ acetate buffer (pH 5.5) with $0.1 \mathrm{mM}$ $\mathrm{H}_{2} \mathrm{O}_{2}$. Versatile peroxidase (VP) activity was determined by the oxidation of $20 \mathrm{mM}$ 2,6-dimethoxyphenols (2,6DMP). Mn-independent activity of VP was estimated by the oxidation of $15 \mu \mathrm{l}$ of $20 \mathrm{mM} 2,6-\mathrm{DMP}\left(\varepsilon_{468}=49.6\right.$ $\left.\mathrm{M}^{-1} \mathrm{~cm}^{-1}\right)$ in $265 \mu \mathrm{l} 50 \mathrm{mM}$ sodium malonate buffer $\left(\mathrm{pH} 3.0\right.$ and 4.5) with $10 \mu \mathrm{l}$ of $6 \mathrm{mM} \mathrm{H}_{2} \mathrm{O}_{2}$. Mndependent activity of VP was assayed by oxidation of $15 \mu \mathrm{l} 20 \mathrm{mM}$ 2,6-DMP in $250 \mu \mathrm{l} 50 \mathrm{mM}$ sodium malonate buffer ( $\mathrm{pH} 4.5)$ in the presence of $10 \mu \mathrm{l}$ of 6 $\mathrm{mM} \mathrm{H}_{2} \mathrm{O}_{2}$ and $15 \mu \mathrm{l} 0.1 \mathrm{mM} \mathrm{MnSO}_{4}$. One unit of specific enzyme activity $\left(\mathrm{U} \mathrm{mg}^{-1}\right)$ was defined as the amount of enzyme that oxidized $1 \mu \mathrm{M}$ of the substrate per minute under defined conditions. The protein concentration was determined according to the Bradford method (1976) [12] using the Protein Assay Kit (BioRad).

\subsection{Phytotoxicity asaay}

The phytotoxicity assay was performed for the untreated medium with $1 \%$ molasses and supernatants obtained after 21-day stationary cultures and 7-day immobilized cultures of $B$. adusta CCBAS 930 and its 930-5 mutant. The phytotoxicity assay was carried out to determine root growth inhibition (I) and germination capacity (GI) in Lepidium sativum. L. seeds before and after decolorization [13]. Petri dishes were lined with filter paper and, subsequently, 100 seeds and $5 \mathrm{ml}$ of the filtered $(\varnothing=0.22 \mu \mathrm{m})$ sample were added. The dishes were incubated at room temperature for 72 hours. Distilled water was used as a control.

\subsection{Biotoxicity assay using resazurin reduction} method
The initial $0.01 \%$ dye solution and post-culture fluids from immobilized cultures of B. adusta CCBAS 930 strain were tested against Gram-positive Staphylococcus aureus ATCC 29737 and Gramnegative Eschericha coli ATCC 25922. The strains were provided by ATCC and stored at $4^{\circ} \mathrm{C}$. All strains were cultured on nutrient broth (NB) medium at $37^{\circ} \mathrm{C}$. Resazurin is a non-toxic water-soluble dye previously applied in bacterial viability studies [14]. This assay is based on detection of the metabolic activity of the cells. The redox dye resazurin (7-hydroxy-3H-phenoxazin-3one 10-oxide) enters the cell in the oxidized form (blue), where it is converted to a reduced form, resorufin (pink). The reduced and oxidized forms of resazurin can be measured separately with a spectrophotometer and used to determine the reduction capability of cells, which reflects the mitochondrial function and cell viability and shows time- and concentration-dependent cell growth inhibition. Serial twofold dilutions of initial $0.01 \%$ dyes solution and post-culture fluids from immobilized cultures of $B$. adusta CCBAS 930 were made with Mueller Hinton Broth (MHB) to yield final concentrations ranging from 10 to $0.62 \mathrm{mg} \mathrm{ml}^{-1}$ (1 $0.062 \%$ ) and placed into a 96-well plate. The bacterial suspension $(100 \mu \mathrm{l})$ prepared from an overnight culture was adjusted to inoculation of $10^{8} \mathrm{CFU} \mathrm{mL}^{-1}$. Then, $100 \mu \mathrm{L}$ of bacterial culture were added. The wells with MHB or yeast culture were the negative and positive control, respectively. The plates were incubated at $37^{\circ} \mathrm{C}$ for $48 \mathrm{~h}$. Then, $20 \mu \mathrm{l}$ of a $60-\mu \mathrm{M}$ resazurin solution in PBS buffer were added to each well. After incubation $\left(2 \mathrm{~h}, 37^{\circ} \mathrm{C}\right)$, the viability of cells was monitored by measuring absorbance at $570 \mathrm{~nm}$ (reduced) and $600 \mathrm{~nm}$ (oxidized) [14] and calculating the resazurin reduction factor (RRF).

\subsection{Data analysis}

The results were presented as a mean of three repetitions for which standard deviation was calculated.

\section{Results}

The results showed that the use of immobilised mycelium of $B$. adusta CCBAS 930 significantly reduces the time of decolorization of $\mathrm{ABBB}, \mathrm{AB} 129$, $\mathrm{CA}$ and RBBR. After 7 days decolorization degree of $\mathrm{CA}, \mathrm{AB} 129, \mathrm{ABBB}$ and RBBR was $75.50 \%, 65.08 \%$, $56.57 \%$ and $25,64 \%$, respectively (Fig. 1 ). 


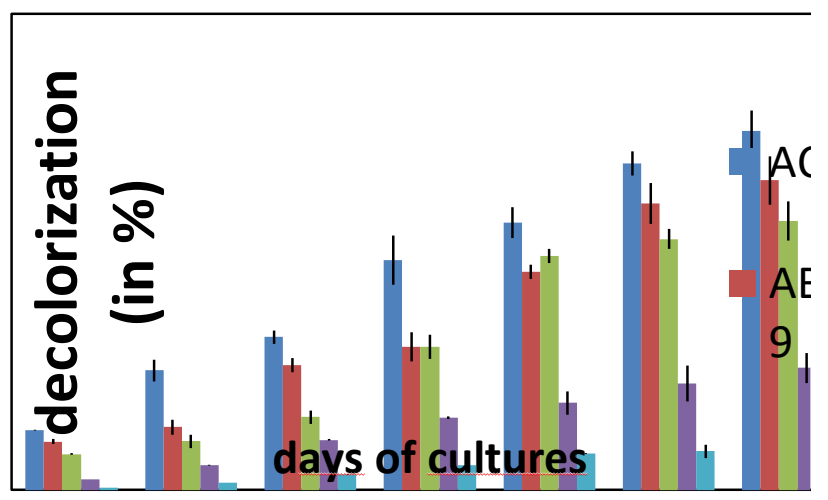

Fig. 1. Decolorization degree of anthraquinone dyes in immobilized cultures of B. adusta CCBAS 930

In immobilised cultures of B. adusta CCBAS 930 with $0.01 \%$ of anthraquinone dyes activity of all tested peroxidases was detected, but HRP-like and VP was characterized by the highest activity. Activity of HRP-like and VP systematically increased reaching the maximum after 7 days, $35.64-80.32$ and $50.20-106.43$ $\mathrm{U} \mathrm{mg}^{-1}$ protein, respectively (Fig.2).
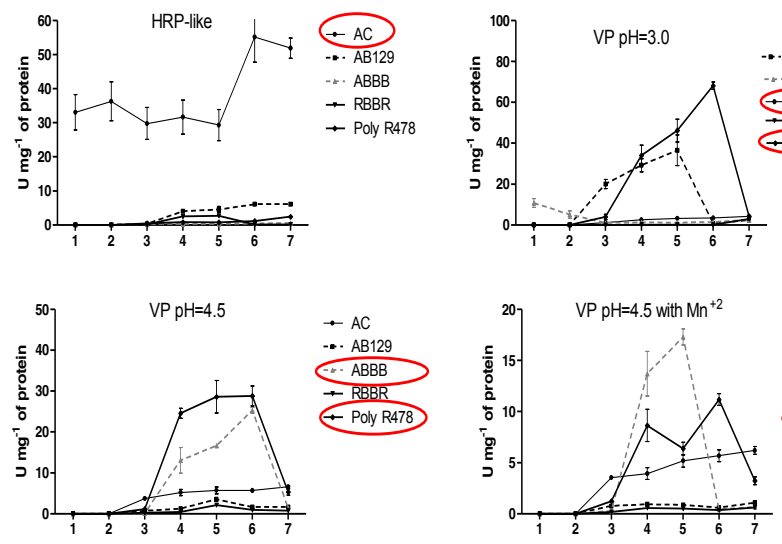

Fig. 2. Activities of peroxidases during immobilized cultures of $B$. adusta CCBAS 930 in the presence of anthraquinone dyes

During the immobilised culture of $B$. adusta CCBAS 930 with $0.01 \%$ anthraquinone dyes, a systematic decrease in the content of phenolic compounds was observed (50-60\%) (Fig. 3).

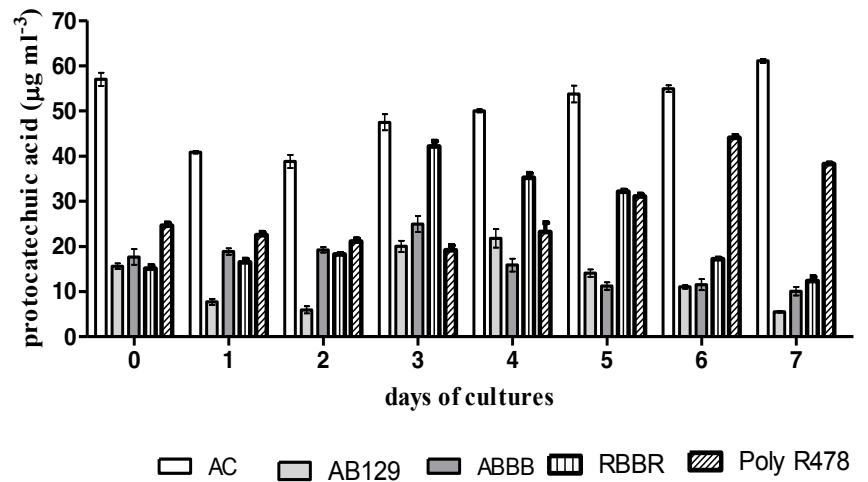

Fig. 3. Content of phenolic compounds during biotransformation of anthraquinone dyes by immobilized B. adusta CCBAS 930

Decolorized post-culture fluids were characterized by lower phyt- and biotoxicity. Decolorized post-culture liquids were characterized by a similar level of inhibition of root growth as control medium initial solution of tested dyes. After biological treatment we observed higher germination index compared to the control solution (Fig.4).

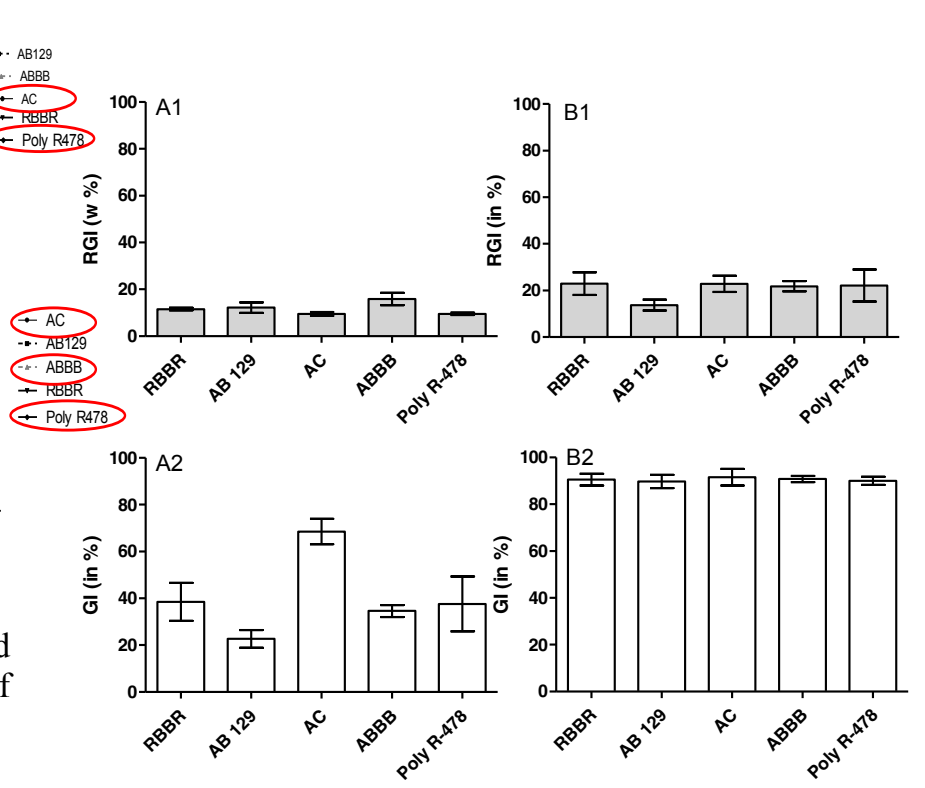

Fig. 4. Root growth inhibition (RGI) and germination index (GI) before (A1, B1) and after decolorization (A2, B2) in cultures of B.adusta CCBAS 930

The biotoxicity assay with Gram-positive and Gramnegative bacteria demonstrated a similar RRF value for the supernatants after immobilized cultures of $B$. adusta CCBAS 930 and control bacteria cultures. Based on these results, it can be concluded that no toxic products are formed during removal of anthraquinone dyes by immobilized B. adusta CCBAS (Fig. 5). 


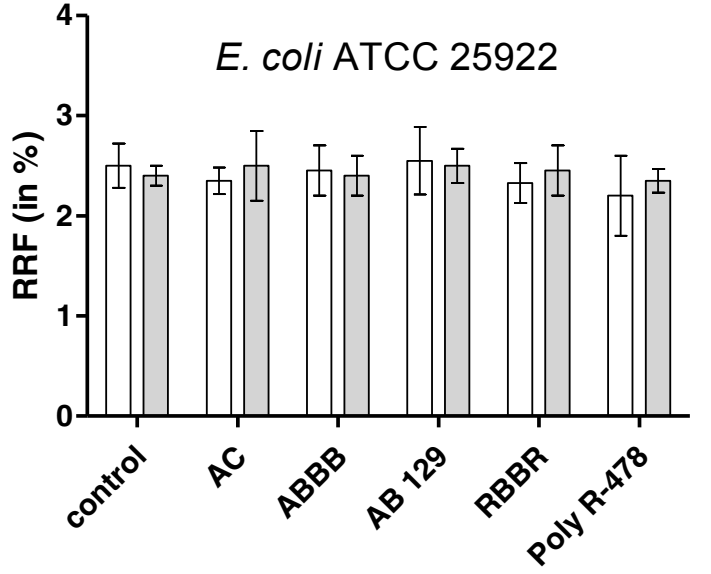

0,01\% anthraquinone dyes after cultures

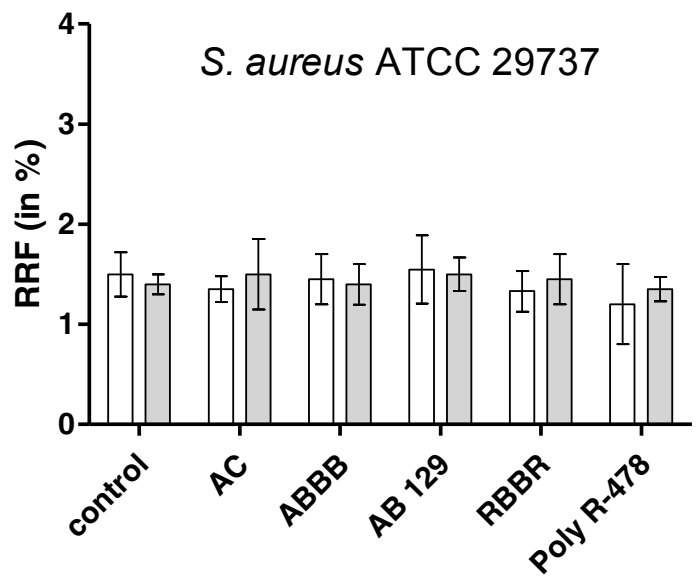

Fig.5. Resazurine reduction factors (RRF) (C,D) of control solutions of $0.01 \%$ anthraquinone dyes and decolorized postculture liquids from immobilized cultures of $B$. adusta CCBAS 930

\section{Discussion}

White rot fungi have the ability to remove dyes of a complex structure, and immobilization of mycelium increase the efficiency of this process, including reduced time of dyes removal [15]. Compared to conventional cultures, the use of immobilized mycelium allows for more effective removal of synthetic anthraquinone dyes and gives the possibility of using a biological method in various industries $[8,16]$. In addition, liquids after immobilized cultures do not show biotoxicity or phytotoxicity as opposed to traditional cultures. In conventional cultures, various metabolites are formed during the growth of the fungus in the presence of synthetic dyes. Secondary products may also be formed which may be toxic [17].

Based on the results obtained in this study, it can be concluded that the removal of anthraquinone dyes using immobilized mycelium of B. adusta CCBAS 930 is more effective and efficient compared to cultivating this strain in stationary conditions. After 7 days, removal of RBBR and Poly R-478 dye was observed at 25\% and $10 \%$, respectively. Studies of Korniłłowicz-Kowalska and Rybczyńska (2012) [4] on removal of $0.01 \%$ RBBR and $0.03 \%$ Poli-R-478 in liquid cultures of $B$. adusta CCBAS 930 showed only a $5 \%$ and $12 \%$ decrease in color medium, respectively. After 18 days of culture, total decolorization of $0.01 \%$ RBBR was noted. In contrast, complete decolorization of polyanthraquinone dye Poly-R 478 was observed on 30 day of culture. Research of Korniłłowicz-Kowalska and Rybczyńska (2014) [5] on the cultivation of strain B. adusta CCBAS 930 with $0.01 \%$ AC under static conditions showed that after 4 days, the basidiomycete removed more than $72 \%$. This corresponded to a $76.82 \%$ reduction in this dye concentration in the medium. The results obtained in this work show that the removal of $0.01 \% \mathrm{AC}$ in the B. adusta CCBAS 930 immobilized culture was not accelerated. In the case of $0.01 \% \mathrm{AC}$, the final effect is similar to the results obtained in B. adusta CCBAS 930 stationary culture. In the research of L'opez et al. (2006) [18] Poly R-478 and RBBR in a concentration of $200 \mathrm{mg}$ $\mathrm{L}^{-1}$, were removed by 31 fungi. In the case of Poly R-478, only 6 strains showed effective, over $90 \%$ decolorization after 7 days. The authors also found that RBBR was decolorized only by three strains LFM-4, LFM-19, LFM-22 at 50\% after 7 days [18]. The results of this study can confirm the above conclusion because RBBR and Poly R-478 dyes from the five analyzed were the most difficult to biodegrade by the immobilized mycelium B. adusta CCBAS 930

Our previous study indicated that in stationary culture of B. adusta with the addition of Poly R-478 and RBBR, a decrease in phenolic compounds noted. A decrease in phenolics was observed after 7 and 10 days of fungal growth [4]. In addition, in the optimized cultures of microscopic fungi $H$. hematococca BwIII43, K37 and T. harzianum BsIII33 with the addition of $0.03 \%$ ABBB dye found that the content of phenolic compounds decreased by $70-76 \%$ and $30 \%$ respectively after 2 weeks [19]. A 43\% drop in phenolic compounds after 7 days of immobilized cultures of $B$. adusta CCBAS 930 with $0.01 \%$ ABBB allows to conclude that the immobilization affects the acceleration of the decrease in phenolic compounds content during ABBB biodegradation. On this basis, the effectiveness of immobilization can be determined compared to stationary culture.

The results of this study show that anthraquinone dyes are removed by the immobilized mycelium B. adusta CCBAS 930 using extracellular peroxidases: HRP-like and (VP). The results of the obtained research are consistent with the previous ones, which describe the decolorization of anthraquinone dyes by fungal peroxidases $[4,5,10]$. The results of the research by Rybczyńska-Tkaczyk et al. (2018) [10] showed that other fungi like $H$. haematococca BwIII43, K37 and $T$. harzianum BsIII33 due to the possibility of synthesizing extracellular peroxidases are able to remove $0.03 \%$ ABBB monoanthraquinone dye. 
This study on the immobilized mycelium B. adusta CCBAS 930 also indicates that the test strain is able to decolorize $0.01 \% \mathrm{ABBB}$ at $56.5 \%$ in 7 days using extracellular peroxidases VP and HRP-like. Our earlier studies show that the stationary cultures of $H$. hematococca BwIII43, $\mathrm{K} 37$ and T. harzianum BsIII33 with the addition of $0.03 \%$ ABBB showed the activity of enzymes such as laccase, LiP, MnP and HRP-like. In the cultures of these mushrooms, the highest HRP-like peroxidase activity was recorded in the first week and it was $106,63-109,47 \mathrm{U}^{*} \mathrm{mg}^{-1}$ [19]. In cultures of $B$. adusta CCBAS 930 with $0.01 \%$ Poly R-478 and AC, maximum HRP-like peroxidase activity was observed on 18 and 21 days $-33,81 \mathrm{mU}^{*} \mathrm{ml}^{-1}$ oraz $13,27 \mathrm{mU}^{*} \mathrm{ml}^{-1}$ respectively [5]. Tinoco et al. (2007) [20] studied the removal of industrial dyes by VP peroxidase from $B$. adusta UAMH 8258 and found significant differences in the dyeing of these dyes. Moreira et al. (2006) [21] isolated VP from Bjerkandera sp B33/3, which effectively removed RBBR dye. According to the authors, VP peroxidase from B33/3 strain has unique properties. Hovewer, they show differences between VP peroxidases synthesized by different Bjerkander strains.

An important aspect of biodegradation is also the toxicity of anthraquinone dyes. Most often, the decrease in the toxicity of synthetic dyes is measured using bacteria and $L$. sativum $\mathrm{L}$. seeds $[10,13]$. The bio- and phytotoxicity tests performed in this study showed that the post-culture liquids of $B$. adusta CCBAS 930 are not toxic to the tested E. coli and S. aureus bacteria. In the case of a phytotoxicity assay, discolored post-culture liquids were characterized by a similar degree of root length inhibition as the initial solutions of the dyes In contrast, the germination index of L. sativum L. seeds was much higher for decolorized post-culture liquids $(88.20 \%$ - 95.21\%) than the initial solutions of anthraquinone dyes $(33.87 \%-72.34 \%)$. Therefore, it can be concluded that no toxic products of their biotransformation were formed during the removal of anthraquinone dyes in immobilized B. adusta CCBAS 930 cultures.

\section{Conclusion}

The results showed that the use of immobilised mycelium of $B$. adusta CCBAS 930 significantly reduces the time of decolorization of anthraquinone dyes. After 7 days decolorization degree of CA, AB129, ABBB and RBBR was $75.50 \%, 65.08 \%, 56.57 \%$ and $25,64 \%$, respectively. Activity of HRP-like and VP peroxidase systematically increased during immobilized cultures with the maximum between 4 and 7 days, 35.64 - 80.32 and $50.20-106.43 \mathrm{U} \mathrm{mg}^{-1}$ protein, respectively. In case of phenolic compounds systematic decrease it content was observed (50-60\%). Decolorized postculture fluids were characterized by lower bio- and phytotoxicity.

\section{Acnowledgements}

Publication is funded by the Polish National Agency for Academic Exchange under the International Academic Partnerships Programme from the project „Organization of the 9th International Scientific and Technical Conference entitled Environmental Engineering, Photogrammetry, Geoinformatics Modern Technologies and Development Perspectives".

\section{References}

1 Q. Huang, R.A. Pinto, K, Griebenow, R., Shweitzer-Stenner, W.J. Weber, J. Am. Chem. Soc. 127 (5), 1431-1437 (2005)

2 Y. Fu, T. Viraraghavan, Bioresour Technol 79, 251-262 (2001)

3 R.C. Kuhad, N. Sood, K.K. Tripathi, A. Singh O.P. Adv. Appl. Microbiol. 56, 185-213 (2004)

4 T. Korniłłowicz-Kowalska, K, Rybczyńska, K Cent. Eur. J. Biol. 7, 948-956 (2012)

5 T. Korniłłowicz-Kowalska, K. Rybczyńska World J. Microbiol. Biotechnol. 30, 1725-1736 (2014)

6 T. Korniłłowicz-Kowalska, H. Iglik, Pol. J. Environ. Stu. 16, 4720-482 (2007)

7 Z. Bouabidi, M. El-Naas, Z. Zhang, Environ. Chem. Lett. 17, 241-257 (2018)

8 M.B. Kurade, T.R. Waghmode, J.Q, Xiong, S.P. Govindwar, B.H. Jeon, 2019. J. Clean. Prod. 213, 884-891 (2019)

9 T. Korniłłowicz-Kowalska, M. Wrzosek, G. Ginalska, H. Iglik, R. Bancerz, Enzyme Microb. Technol. 38, 583-590 (2006)

10 K. Rybczyńska-Tkaczyk, A. Święciło, K.A. Szychowki, T. Korniłłowicz-Kowalska. Ecotox. Environ. Safe. 147, 776-787 (2018)

11 A. Święciło, K. Rybczyńska-Tkaczyk, A. Najda, A., Krzepiłko, R Prażak, G Zawiślak, G., LWT Food Sci. Technol. 94, 96-102 (2018)

12 M.M. Bradford, Anal. Biochem. 7, 248- 254 (1976)

13 K. Rybczyńska-Tkaczyk, T. KorniłłowiczKowalska, Int. Biodeterior. Biodegrad. 117,131-140 (2017)

14 I Osaka, S. Hefty, Antimicrob. Agents Chemother. 57, 2838-2840 (2013)

15 A. Dominguez, S.R. Couto, M.A. Sanromán, World J. Microbiol. Biotechnol. 21, 405-409 (2005)

16 B.B. Hameed, Z.Z. Ismail, Biochem. Eng. J. 137, 71-77 (2018)

17 E. Forgacs, T. Cserha'ti, G. Oros G Removal of synthetic dyes from wastewaters: a review. Environ Int 30:953-971 
18 M.J. Lopez, G. Guisado, M.C. Vargas-Garcia, F. Sua rez-Estrella, J. Moreno, Enzyme. Microb. Technol. 40, 42-45 (2006)

19 T. Korniłłowicz-Kowalska, K. Rybczyńska, Int. J. Environ. Sci. Technol. 12, 2673-2686 (2015)

20 R. Tinoco, J. Verdin, R. Vazquez-Duhalt, J. Mol. Cat. B: Enzym. 46, 1-7 (2007)

21 P.R. Moreira, F. Bouillenne, E. Almeida-Vara, F. Xavier Malcata, J.M. Frére, J.C. Duarte, Enzyme Microb. Technol. 38, 28-3 (2006) 